\title{
An Improved Internal Model Principle Based Multivariable Nonlinear Control Method with Multiclass Nonharmonic Disturbances and Its Application to Speed Control of a Motor Drive System
}

\author{
Yang Yu, Zengqiang Mi, Tong Zhao, and Yikun Xu \\ State Key Laboratory of Alternate Electrical Power System with Renewable Energy Sources, North China Electric Power University, \\ Baoding 071003, China \\ Correspondence should be addressed to Yang Yu; ncepu_yy@163.com
}

Received 17 April 2014; Revised 17 May 2014; Accepted 31 May 2014; Published 19 June 2014

Academic Editor: Shen Yin

Copyright (C) 2014 Yang Yu et al. This is an open access article distributed under the Creative Commons Attribution License, which permits unrestricted use, distribution, and reproduction in any medium, provided the original work is properly cited.

\begin{abstract}
We study the global disturbance rejection problem for a class of general multivariable nonlinear systems with multiclass nonharmonic disturbances. The paper first introduces the importance and state of the art for disturbance rejection problem and describes the control problem in the form of mathematical expressions. It stresses the multiclass disturbances produced by the exosystem satisfying certain characteristic conditions. Then, the nonlinear internal models are designed in accordance with different characteristics of multiclass external disturbances. On the basis of introduction of the control law for disturbance-free system, a multivariable state feedback controller is devised in terms of the designed internal model equations and corresponding assumptions. A Lyapunov function is constructed to theoretically prove the global uniform boundness of all signals for the multivariable closedloop system. Finally, the presented method is applied to implement the speed control and reject the multiclass nonharmonic disturbances for a two-input motor drive system. The simulation results testify correctness and effectiveness of the presented algorithm.
\end{abstract}

\section{Introduction}

Due to its importance, the problem of disturbance rejection generated by the exosystem has been paid great attention in the world over the past few decades [1-3]. Especially, this problem in nonlinear system has been concerned in a recent period of time [4-6]. In terms of internal model principle (IMP), one of the key technologies of rejection of exogenous disturbance is to construct reasonable internal model equations, which is able to duplicate the characteristics of the external disturbance.

In addition, many industrial applications, such as servo system, wind power system, photovoltaic system, and other modern systems, which rely on power electronic equipments to work, [7-9], are frequently influenced by the external disturbances generated by exosystem $[10,11]$. In the circumstance of the existence of external disturbances, the conventional PID controller with constant parameters is difficult to satisfy the requirement of high precision control for these systems $[12,13]$. Therefore, rejection of external disturbances by means of new ways $[14,15]$ is an important work to improve the working performance of these industrial systems. From the state-of-the-art technologies in disturbance rejection, the researchers frequently assume the following:

(1) the external disturbances are frequently sinusoidal; that is to say, the exosystem generally satisfies the condition of neutral stability such as references $[16,17]$ discussing the suppression of sinusoidal interference with known and unknown frequency, respectively;

(2) currently, the focus of the research on disturbance rejection is gradually converting from linear systems to nonlinear systems; nevertheless, the disturbance rejections with semiglobal stability [18] and singleinput and single-output (SISO) [19] systems are concerned. 
Therefore, the disturbance rejection problem with nonharmonic interferences and multivariable nonlinear systems has not received adequate attention in current studies. However, the nonharmonic disturbances produced by nonlinear exosystem can induce noise and reduce accuracy to these industrial systems [20]. In addition, just a few articles on nonharmonic interference suppression aim at one class of disturbance [21]. Therefore, the research of multiclass disturbances rejection in multivariable nonlinear system can extend the application of present control theory to a generalized range [22]. Hence, in [23], a global multivariable control algorithm is proposed to reject multiclass external nonharmonic disturbances.

In view of the issues described above, the purpose of the paper is to propose an improved internal model principle (IMP) based multivariable nonlinear control algorithm with multiclass nonharmonic disturbances and utilize the proposed method to control a motor drive system to run stably and suppress multiclass external nonharmonic disturbances. As written in [23], the critical points of the problem in disturbance rejection are to model the nonlinear exosystem and present a corresponding control method to suppress the disturbance. Therefore, the essential distinction with the work in [23], this paper addresses the problem of multivariable disturbances rejection with other multiclass exosystems, additionally, which are provided by matrix form. For the difference of external exosystems, the entirely different internal model and a matching control method should be proposed to model and reject the external disturbances in terms of the intrinsic characteristics of exosystem. In practical terms, the construction of internal model replicating with the exogenous interference in the paper is on the basis of selfcontained concepts of expanded steady state generator and internal model proposed in [24]. Similarly, the problem of nonharmonic disturbances rejection in the paper is different from the work in [22], which aims to reject a constant disturbance.

The innovative works in the paper are the following:

(1) the previous work in references $[20,21]$ is extended to a generalized field with multivariable inputs of nonlinear systems and multiclass nonlinear exosystems;

(2) an improved IMP based multivariable nonlinear control algorithm with another multiclass nonharmonic disturbance is presented on the basis of certain characteristic conditions satisfied by exosystem and state information of closed-loop system;

(3) a new type of nonlinear multivariable internal models for another multiclass nonlinear nonharmonic disturbance is constructed in terms of the expanded steady state generator and internal model.

The structures of the paper are arranged as follows. It begins in Section 1 with an analysis of the state of the art of disturbance rejection. The formulation of control problem in the paper is given in Section 2. The nonlinear internal models of multiclass external disturbances are designed in Section 3. The nonlinear multivariable state feedback controller is devised in Section 4, and the global convergence of the presented controller is theoretically proved. The implementation of the proposed algorithm to a two-input motor drive system is performed in Section 5 and the conclusions of the research are obtained in the last section.

\section{Formulation of Control Problem}

The multivariable nonlinear system with multiclass external nonharmonic disturbances studied in the paper is described in an affine form shown as below [23]:

$$
\dot{\mathbf{x}}=\mathbf{f}(\mathbf{x})+\sum_{i=1}^{m} \mathbf{g}_{i}(\mathbf{x})\left(\mathbf{u}_{i}+\mathbf{v}_{i}(\mathbf{w})\right), \quad 1 \leq i \leq m,
$$

where $\mathbf{x} \in \mathbf{R}^{n}$ indicate the state vectors, $\mathbf{u}_{i} \in \mathbf{R}$ describe the control inputs, $\mathbf{f}(\mathbf{x})$ and $\mathbf{g}_{i}(\mathbf{x})$ denote the known smooth vector fields, $\mathbf{v}_{i}(\mathbf{w})$ represent the external nonlinear disturbances, $\mathbf{w} \in \mathbf{R}^{q}$ are the exogenous vectors generated by the nonlinear exosystem and shown as follows [24]:

$$
\dot{\mathbf{w}}=s_{i}(\mathbf{w})=A_{i 1} \mathbf{w}+\sum_{k=2}^{n} A_{i k} \mathbf{w} s_{i k}(\mathbf{w}), \quad 1 \leq i \leq m,
$$

where matrices $A_{i 1}$ and $A_{i k} \in R^{n_{q} \times n_{q}}, k=2, \ldots, n$, and $s_{i k}(\mathbf{w}): R^{n_{q}} \rightarrow R$ are sufficient smooth functions, and $s_{i k}(0)=0$.

For the stability problems of multivariable input system, the key of the study is to convert multivariable nonlinear system into multiple single-input systems [25].

Assumption 1. For the multivariable nonlinear disturbancefree system shown below:

$$
\dot{\mathbf{x}}=\mathbf{f}(\mathbf{x})+\sum_{i=1}^{m} \mathbf{g}_{i}(\mathbf{x}) \mathbf{u}_{i}, \quad 1 \leq i \leq m,
$$

there exists control law of sate feedback $\boldsymbol{\alpha}_{i}(\mathbf{x})$, which can make the system

$$
\dot{\mathbf{x}}=\mathbf{f}(\mathbf{x})+\sum_{i=1}^{m} \mathbf{g}_{i}(\mathbf{x}) \boldsymbol{\alpha}_{i}(\mathbf{x})
$$

asymptotically converge to the origin. In consequence, a Lyapunov function $\mathbf{V}(\mathbf{x})$ exists and makes the following inequalities hold $[20,23]$ :

$$
\begin{gathered}
\underline{d}(\|\mathbf{x}\|) \leq \mathbf{V}(\mathbf{x}) \leq \bar{d}(\|\mathbf{x}\|) \\
\frac{\partial \mathbf{V}(\mathbf{x})}{\partial \mathbf{x}}\left(\mathbf{f}(\mathbf{x})+\sum_{i=1}^{m} \mathbf{g}_{i}(\mathbf{x}) \boldsymbol{\alpha}_{i}(\mathbf{x})\right) \leq-d_{0}(\|\mathbf{x}\|), \\
\left|\frac{\partial \mathbf{V}(\mathbf{x})}{\partial \mathbf{x}} \sum_{i=1}^{m} \mathbf{g}_{i}(\mathbf{x})\right|^{2} \leq d_{0}(\|\mathbf{x}\|)
\end{gathered}
$$

where $\underline{d}, \bar{d}$, and $d_{0}$ belong to class $K_{\infty}$ functions.

Assumption 2. The flows of the vector field for nonlinear exosystem (2) are bounded. 
Remark 3. Generally speaking, there exist numerous nonlinear systems satisfying Assumption 2, such as harmonic functions and limit cycles of nonlinear dynamic system. The well-known Van der Pol oscillator [20] is selected as the exosystem in the paper and can be expressed as below:

$$
\begin{aligned}
& \dot{w}_{1}=w_{2}, \\
& \dot{w}_{2}=-a w_{1}+b\left(1-w_{1}^{2}\right) w_{2},
\end{aligned}
$$

where $a$ and $b$ are constants greater than zero. Under such circumstances, the limit cycle generated by Van der Pol oscillator is stable. Consequently, (6) is written in the form of (2), and we can obtain

$$
\dot{\mathbf{w}}=A_{11} \mathbf{w}+A_{12} \mathbf{w} s_{12}(\mathbf{w})
$$

where $A_{11}=\left[\begin{array}{cc}0 & 1 \\ -a & b\end{array}\right], A_{12}=\left[\begin{array}{cc}0 & 0 \\ 0 & -b\end{array}\right], s_{12}(w)=w_{1}^{2}$.

Assumption 4. There exist a constant $r_{i}$ and a set of real numbers $a_{i 0}, a_{i 1}, \ldots, a_{i\left(r_{i}-1\right)}$ satisfying the following equation:

$$
\begin{aligned}
L_{A_{i 1} \mathbf{w}}^{r_{i}} \mathbf{v}_{i}(\mathbf{w})= & a_{i 0} \mathbf{v}_{i}(\mathbf{w})+a_{i 1} L_{A_{i 1} \mathbf{w}} \mathbf{v}_{i}(\mathbf{w}) \\
& +\cdots+a_{i\left(r_{i}-1\right)} L_{A_{i 1} \mathbf{w}}^{r_{i}-1} \mathbf{v}_{i}(\mathbf{w}),
\end{aligned}
$$

where $L_{A_{i 1} \mathbf{w}} \mathbf{v}_{i}(\mathbf{w})=\left(\partial \mathbf{v}_{i}(\mathbf{w}) / \partial \mathbf{w}\right) A_{i 1} \mathbf{w}, L_{A_{i 1} \mathbf{w}}^{m} \mathbf{v}_{i}(\mathbf{w})=\left(\partial L_{A_{i 1} \mathbf{w}}^{m-1}\right.$ $\left.\mathbf{v}_{i}(\mathbf{w}) / \partial \mathbf{w}\right) A_{i 1} \mathbf{w}, m=2,3, \ldots, r_{i}$, and $L$ is an operator of Lee derivative.

Apparently, if $\mathbf{v}_{i}(\mathbf{w})$ denotes a linear expression of $\mathbf{w}$, Assumption 4 will hold automatically.

Assumption 5. There exists a smooth function $\mathbf{h}_{i}(\mathbf{x}): \mathbf{R}^{n} \rightarrow$ $\mathbf{R}^{n_{q}}$ making

$$
\frac{\partial \mathbf{h}_{i}(\mathbf{x})}{\partial \mathbf{x}} \mathbf{g}_{i}(\mathbf{x})=\mathbf{G}_{i}, \quad 1 \leq i \leq m
$$

where $\mathbf{G}_{i}$ is a nonzero constant vector defined in $\mathbf{R}^{n_{q}}$. below.

The questions resolved in the paper can be depicted as

Definition 6. For an arbitrarily given compact subset $\mathbf{D}_{\mathbf{w}} \in$ $\mathbf{R}^{n_{q}}$, a state feedback controller $\mathbf{u}_{i}$ can always be found to guarantee the solution of original multivariable closed-loop system (1) existence and boundness under arbitrary initial conditions for all $\mathbf{w}(\mathbf{0}) \in \mathbf{D}_{\mathbf{w}}$, when $t \geq 0$, and even $\lim _{t \rightarrow \infty} \mathbf{x}(t)=\mathbf{C}$, where $\mathbf{C}$ is a constant vector representing the reference value.

\section{Design of Multiclass Nonlinear Internal Models}

Let

$$
\tau_{i}(\mathbf{w})=\operatorname{col}\left(\mathbf{v}_{i}(\mathbf{w}), L_{A_{i 1} \mathbf{w}} \mathbf{v}_{i}(\mathbf{w}), \ldots, L_{A_{i 1} \mathbf{w}}^{r_{i}-1} \mathbf{v}_{i}(\mathbf{w})\right) ;
$$

then there exist matrices $\Phi_{i}=\left[\begin{array}{cc}0_{\left(r_{i}-1\right) \times 1} & I_{r_{i}-1} \\ a_{i 0} & {\left[a_{i 1}, \ldots, a_{r_{i}-1}\right.}\end{array}\right]$ and $\psi_{i}=$ $[1,0, \ldots, 0]$ making

$$
\begin{gathered}
\frac{\partial \tau_{i}(\mathbf{w})}{\partial \mathbf{w}} A_{i 1} \mathbf{w}=\Phi_{i} \tau_{i}(\mathbf{w}), \\
v_{i}(\mathbf{w})=\psi_{i} \tau_{i}(\mathbf{w}),
\end{gathered}
$$

where matrix pair $\left(\Phi_{i}, \psi_{i}\right)$ is observable and $I_{r_{i}-1}$ is $\left(r_{i}-1\right) \times$ $\left(r_{i}-1\right)$ unit matrix.

For the sake of establishment of nonlinear internal model equation, the following assumption is brought up.

Assumption 7. There exists a matrix $\Phi_{i k}, k=2, \ldots, n$, making

$$
\frac{\partial \tau_{i}(\mathbf{w})}{\partial \mathbf{w}} A_{i k} \mathbf{w}=\Phi_{i k} \tau_{i}(\mathbf{w})
$$

Assume $\widehat{\tau}_{i}(\mathbf{w})=T_{i} \tau_{i}(\mathbf{w})$ and $T_{i} \in R^{r_{i} \times r_{i}}$ to be nonsingular matrices. With derivative of $\widehat{\tau}_{i}(\mathbf{w})$ along with (2), we can obtain

$$
\begin{aligned}
\frac{\partial \widehat{\tau}_{i}(\mathbf{w})}{\partial \mathbf{w}} s_{i}(\mathbf{w}) & =\frac{\partial \widehat{\tau}_{i}(\mathbf{w})}{\partial \mathbf{w}}\left(A_{i 1} \mathbf{w}+\sum_{k=2}^{n} A_{i k} \mathbf{w} s_{i k}(\mathbf{w})\right) \\
& =T_{i} \Phi_{i} T_{i}^{-1} \widehat{\tau}_{i}(\mathbf{w})+\sum_{k=2}^{n} T_{i} \Phi_{i k} s_{i k}(\mathbf{w}) T_{i}^{-1} \widehat{\tau}_{i}(\mathbf{w}) \\
& =T_{i} \phi_{i}(w) T_{i}^{-1} \widehat{\tau}_{i}(\mathbf{w}) \\
v_{i}(\mathbf{w}) & =\psi_{i} T_{i}^{-1} \widehat{\tau}_{i}(\mathbf{w})
\end{aligned}
$$

where $\phi_{i}(\mathbf{w})=\Phi_{i}+\varphi_{i}(\mathbf{w}), \varphi_{i}(\mathbf{w})=\sum_{k=2}^{n} \Phi_{i k} s_{i k}(\mathbf{w})$, and $\varphi_{i}(0)=0, \phi_{i}(0)=\Phi_{i}$.

In terms of linear observer theory, a Hurwitz matrix $\mathbf{F}_{i}$ is chosen to make matrix pair $\left(\mathbf{F}_{i}, \mathbf{G}_{i}\right)$ be controllable for nonzero constant vector $G_{i}$ defined in (9). Due to the observability of matrix pair $\left(\Phi_{i}, \psi_{i}\right)$ and the fact that $\mathbf{F}_{i}$ and $\Phi_{i}$ have nonintersecting frequency spectrum, hence Sylvester equation $T_{i} \Phi_{i}-F_{i} T_{i}=G_{i} \psi_{i}$ has a unique nonsingular solution $T_{i}$.

Let $q_{i}=\psi_{i} T_{i}^{-1}$, the nonlinear exosystem can immerse into the system shown as below:

$$
\begin{gathered}
\dot{\boldsymbol{\eta}}_{i}=\left(\mathbf{F}_{i}+T_{i} \varphi_{i}(\mathbf{w}) T_{i}^{-1}\right) \boldsymbol{\eta}_{i}+\mathbf{G}_{i} q_{i} \boldsymbol{\eta}_{i} ; \\
\mathbf{v}_{i}(\mathbf{w})=q_{i} \boldsymbol{\eta}_{i} .
\end{gathered}
$$

In consequence, multiclass nonlinear internal models can be designed as follows:

$$
\begin{aligned}
\dot{\xi}_{i}= & \left(\mathbf{F}_{i}+T_{i} \varphi_{i}(\mathbf{w}) T_{i}^{-1}\right)\left(\xi_{i}+\mathbf{h}_{i}(\mathbf{x})\right) \\
& -\frac{\partial \mathbf{h}_{i}(\mathbf{x})}{\partial \mathbf{x}}\left(\mathbf{f}_{i}(\mathbf{x})+\mathbf{g}_{i}(\mathbf{x}) \mathbf{u}_{i}\right) .
\end{aligned}
$$

Define an auxiliary error $\mathbf{e}_{i}$ as

$$
\mathbf{e}_{i}=\boldsymbol{\eta}_{i}-\xi_{i}-\mathbf{h}_{i}(\mathbf{x}),
$$


and with derivative of (16) along with (1), (14), and (15), we can get

$$
\begin{aligned}
\dot{\mathbf{e}}_{i}= & \left(\mathbf{F}_{i}+T_{i} \varphi_{i}(\mathbf{w}) T_{i}^{-1}\right) \boldsymbol{\eta}_{i}+\mathbf{G}_{i} q_{i} \boldsymbol{\eta}_{i} \\
& -\left(\mathbf{F}_{i}+T_{i} \varphi_{i}(\mathbf{w}) T_{i}^{-1}\right)\left(\xi_{i}+\mathbf{h}_{i}(\mathbf{x})\right) \\
& +\frac{\partial \mathbf{h}_{i}(\mathbf{x})}{\partial \mathbf{x}}\left(\mathbf{f}_{i}(\mathbf{x})+\mathbf{g}_{i}(\mathbf{x}) \mathbf{u}_{i}\right) \\
& -\frac{\partial \mathbf{h}_{i}(\mathbf{x})}{\partial \mathbf{x}}\left(\mathbf{f}_{i}(\mathbf{x})+\mathbf{g}_{i}(\mathbf{x})\left(\mathbf{u}_{i}+\mathbf{v}_{i}(\mathbf{w})\right)\right) \\
= & \left(\mathbf{F}_{i}+T_{i} \varphi_{i}(\mathbf{w}) T_{i}^{-1}\right)\left(\eta_{i}-\xi_{i}-\mathbf{h}_{i}(\mathbf{x})\right) \\
= & \left(\mathbf{F}_{i}+T_{i} \varphi_{i}(\mathbf{w}) T_{i}^{-1}\right) \mathbf{e}_{i} .
\end{aligned}
$$

\section{Design of Multivariable Nonlinear State Feedback Controller}

On the basis of the established multiclass nonlinear internal models (15) and Assumption 1, the multivariable nonlinear state feedback controller can be designed as follows:

$$
\mathbf{u}_{i}=\boldsymbol{\alpha}_{i}(\mathbf{x})-\mathbf{q}_{i}\left(\xi_{i}+\mathbf{h}_{i}(\mathbf{x})\right)
$$

Construct a Lyapunov function $W$ as below:

$$
W=V(\mathbf{x})+\sum_{i=1}^{m} \gamma \mathbf{e}_{i}^{T} \mathbf{e}_{i}
$$

where $\gamma$ is a positive real constant. With derivative of function $W$ along with originally nonlinear system (1) and auxiliary error (17), we can obtain

$$
\begin{aligned}
\dot{W}= & \frac{\partial V(\mathbf{x})}{\partial \mathbf{x}}\left(\mathbf{f}(\mathbf{x})+\sum_{i=1}^{m} \mathbf{g}_{i}(\mathbf{x})\left(\mathbf{u}_{i}+\mathbf{v}_{i}(\mathbf{w})\right)\right) \\
& +\sum_{i=1}^{m}\left(\gamma \mathbf { e } _ { i } ^ { T } \left(\mathbf{F}_{i}+T_{i} \varphi_{i}(\mathbf{w}) T_{i}^{-1}+\mathbf{F}_{i}^{T}\right.\right. \\
& \left.\left.+T_{i}^{-T} \varphi_{i}^{T}(\mathbf{w}) T_{i}^{T}\right) \mathbf{e}_{i}\right) \\
= & \frac{\partial V(\mathbf{x})}{\partial \mathbf{x}}\left(\mathbf{f}_{i}(\mathbf{x})\right. \\
& \left.+\sum_{i=1}^{m} \gamma \mathbf{e}_{i}^{T}(S(\mathbf{w}))\left(\mathbf{\alpha}_{i}(\mathbf{x})-\mathbf{q}_{i}\left(\xi_{i}+\mathbf{h}_{i}(\mathbf{x})\right)+\mathbf{q}_{i} \eta_{i}\right)\right) \\
= & \frac{\partial V(\mathbf{x})}{\partial \mathbf{x}}\left(\mathbf{f}_{i}(\mathbf{x})+\sum_{i=1}^{m} \mathbf{g}_{i}(\mathbf{x}) \boldsymbol{\alpha}_{i}(\mathbf{x})\right)
\end{aligned}
$$

$$
\begin{aligned}
& +\frac{\partial V(\mathbf{x})}{\partial \mathbf{x}} \sum_{i=1}^{m} \mathbf{g}_{i}(\mathbf{x}) \mathbf{q}_{i} \mathbf{e}_{i}+\sum_{i=1}^{m} \gamma \mathbf{e}_{i}^{T}(S(\mathbf{w})) \mathbf{e}_{i} \\
\leq & -d_{0}(\|\mathbf{x}\|)+\left|\frac{\partial V(\mathbf{x})}{\partial \mathbf{x}} \sum_{i=1}^{m} \mathbf{g}_{i}(\mathbf{x})\right|\left\|\mathbf{q}_{i} \mathbf{e}_{i}\right\| \\
& +\sum_{i=1}^{m} \gamma \mathbf{e}_{i}^{T}(S(\mathbf{w})) \mathbf{e}_{i},
\end{aligned}
$$

where $S(\mathbf{w})=\mathbf{F}_{i}+T_{i} \varphi_{i}(\mathbf{w}) T_{i}^{-1}+\mathbf{F}_{i}^{T}+T_{i}^{-T} \varphi_{i}^{T}(\mathbf{w}) T_{i}^{T}$. By application of inequality $2 a b \leq c a^{2}+c^{-1} b^{2}$ ( choose $c=1$ ) into the second item of (20), we can get

$$
\begin{aligned}
& \left|\frac{\partial V(\mathbf{x})}{\partial \mathbf{x}} \sum_{i=1}^{m} \mathbf{g}_{i}(\mathbf{x})\right|\left\|\mathbf{q}_{i} \mathbf{e}_{i}\right\| \\
& \leq \frac{1}{2}\left|\frac{\partial V(\mathbf{x})}{\partial \mathbf{x}} \sum_{i=1}^{m} \mathbf{g}_{i}(\mathbf{x})\right|^{2}+\sum_{i=1}^{m} \frac{1}{2}\left\|\mathbf{q}_{i}\right\|^{2}\left\|\mathbf{e}_{i}\right\|^{2} \\
& \quad \leq \frac{1}{2} d_{0}(\|\mathbf{x}\|)+\sum_{i=1}^{m} \frac{1}{2}\left\|\mathbf{q}_{i}\right\|^{2}\left\|\mathbf{e}_{i}\right\|^{2} .
\end{aligned}
$$

Assume that there exists a compact set $\bar{S}(\mathbf{w})$ making $S(\mathbf{w})$ be a symmetric matrix of negative definiteness for all $\mathbf{w} \epsilon$ $\bar{S}(\mathbf{w})$. Hence, there exists a positive real number $f$ satisfying the following inequality for all $\mathbf{w} \in \bar{S}(\mathbf{w})$ :

$$
\mathbf{e}_{i}^{T}(S(\mathbf{w})) \mathbf{e}_{i} \leq-f\left\|\mathbf{e}_{i}\right\|^{2} .
$$

Substitute (21) and (22) into (20); we can get

$$
\begin{aligned}
\dot{W} \leq & -d_{0}(\|\mathbf{x}\|)+\left|\frac{\partial V(\mathbf{x})}{\partial \mathbf{x}} \sum_{i=1}^{m} \mathbf{g}_{i}(\mathbf{x})\right|\left\|\mathbf{q}_{i} \mathbf{e}_{i}\right\| \\
& +\sum_{i=1}^{m} \gamma \mathbf{e}_{i}^{T}(S(\mathbf{w})) \mathbf{e}_{i} \\
\leq & -d_{0}(\|\mathbf{x}\|)+\frac{1}{2} d_{0}(\|\mathbf{x}\|)+\sum_{i=1}^{m} \frac{1}{2}\left\|\mathbf{q}_{i}\right\|^{2}\left\|\mathbf{e}_{i}\right\|^{2} \\
& -\sum_{i=1}^{m} \gamma f\left\|\mathbf{e}_{i}\right\|^{2} \\
\leq & -\frac{1}{2} d_{0}(\|\mathbf{x}\|)+\sum_{i=1}^{m}\left(\frac{1}{2}\left\|\mathbf{q}_{i}\right\|^{2}-\gamma f\right)\left\|\mathbf{e}_{i}\right\|^{2} .
\end{aligned}
$$

Choose appropriate $\gamma$ and $f$ to satisfy

$$
\theta=\frac{1}{2}\left\|\mathbf{q}_{i}\right\|^{2}-\gamma f<0 ;
$$


then, we can obtain

$$
\dot{W} \leq-\frac{1}{2} d_{0}(\|\mathbf{x}\|)+\sum_{i=1}^{m} \theta\left\|\mathbf{e}_{i}\right\|^{2}<0 .
$$

Hence, all variables are bounded. Combined with the utilization of invariant set theorem, we can get $\lim _{t \rightarrow \infty} \mathbf{x}(t)=\mathbf{C}$ and $\lim _{t \rightarrow \infty} \mathbf{e}_{i}=0$. The following theorem can be obtained.

Theorem 8. For the multivariable nonlinear system (1) and nonlinear exosystem (2) satisfying Assumption 1 to Assumption 7, multiclass nonlinear internal models (15) and multivariable nonlinear state feedback controller (18) are able to make the closed-loop system globally bounded, and $\lim _{t \rightarrow \infty} \mathbf{x}(t)=\mathbf{C}$.

\section{Application to Speed Control of a Two-Input Nonlinear System}

Speed control of a permanent magnet synchronous motor drive system is widely utilized in various industrial fields. Hence, consider a two-input variable motor drive system shown as below [25], which is not able to tackle with a singleinput method of previous disturbance rejection:

$$
\begin{aligned}
\frac{\mathrm{d} i_{d}}{\mathrm{~d} t} & =-\frac{R_{s}}{L_{d}} i_{d}+p i_{q} \omega_{m}+\frac{1}{L_{d}} u_{d}, \\
\frac{\mathrm{d} \omega_{m}}{\mathrm{~d} t} & =\frac{p \varphi_{f}}{J_{m}} i_{q}-\frac{B_{m}}{J_{m}} \omega_{m}-\frac{1}{J_{m}} T_{L}, \\
\frac{\mathrm{d} i_{q}}{\mathrm{~d} t} & =-\frac{R_{s}}{L_{q}} i_{q}-p i_{d} \omega_{m}-\frac{p \varphi_{f}}{L_{q}} \omega_{m}+\frac{1}{L_{q}} u_{q},
\end{aligned}
$$

where $i_{d}$ and $i_{q}$ represent the current in $d$-axis and $q$-axis, respectively, $u_{d}$ and $u_{q}$ indicate the voltage in $d$-axis and $q$ axis, respectively, $R_{s}$ and $\varphi_{f}$ denote the resistance of stator and flux linkage of permanent magnet, $\omega_{m}$ is the speed, $p$ and $J_{m}$ denote number of pole pairs and moment of inertia of rotor, respectively, and $T_{L}$ is an increasing load.

With conversion of (26) into an affine form as (1), we can get

$$
\dot{\mathbf{x}}=\mathbf{f}(\mathbf{x})+\sum_{i=1}^{2} \mathbf{g}_{i}(\mathbf{x})\left(\mathbf{u}_{i}+\mathbf{v}_{i}(\mathbf{w})\right)
$$

where $\mathbf{x}=\left[\begin{array}{lll}x_{1} & x_{2} & x_{3}\end{array}\right]^{T}=\left[\begin{array}{lll}i_{d} & \omega_{m} & i_{q}\end{array}\right]^{T}$,

$$
\begin{gathered}
\mathbf{f}(\mathbf{x})=\left[\begin{array}{c}
-\frac{R_{s}}{L_{d}} x_{1}+p x_{2} x_{3} \\
\frac{p \varphi_{f}}{J_{m}} x_{3}-\frac{B_{m}}{J_{m}} x_{2}-\frac{1}{J_{m}} T_{L} \\
-\frac{R_{s}}{L_{q}} x_{3}-p x_{2} x_{1}-\frac{p \varphi_{f}}{L_{q}} x_{2}
\end{array}\right], \\
\mathbf{g}_{1}(\mathbf{x})=\left[\begin{array}{ll}
\frac{1}{L_{d}} & 0
\end{array}\right]^{T}, \quad \mathbf{g}_{2}(\mathbf{x})=\left[\frac{1}{L_{q}}\right],
\end{gathered}
$$

and control inputs $u=\left[\begin{array}{ll}u_{1} & u_{2}\end{array}\right]^{T}=\left[\begin{array}{ll}u_{d} & u_{q}\end{array}\right]^{T}$.
For the sake of understandability, the external disturbances inputs, $v_{1}$ and $v_{2}$, which denote different nonlinear nonharmonic disturbance input signals, are both produced by Van der Pol oscillator shown in (6), and let $a=b=1$. Hence, $A_{11}=\left[\begin{array}{cc}0 & 1 \\ -1 & 1\end{array}\right], A_{12}=\left[\begin{array}{cc}0 & 0 \\ 0 & -1\end{array}\right]$. Evidently, $A_{21}=A_{11}$ and $A_{22}=A_{12}$. It is important to note that $A_{11}, A_{21}, A_{12}$, and $A_{22}$ are not elements in matrix $A_{1}$ or $A_{2}$, which are all independent matrices defined in (2). For the sake of convenience, let $A_{11}=A_{21}=A_{1}, A_{12}=A_{22}=A_{2}$, and $s_{12}(\mathbf{w})=s_{22}(\mathbf{w})=w_{1}^{2}$. The bounded limit cycle will be generated by the oscillator; hence, Assumption 2 holds.

Let $c_{1}, c_{2}$, and $c_{3}$ all be certain positive constants; the control law of disturbance-free system for (27) can be obtained by means of backstepping control and can be shown as follows:

$$
\begin{aligned}
\boldsymbol{\alpha}(\mathbf{x}) & =\left[\begin{array}{l}
\boldsymbol{\alpha}_{1}(\mathbf{x}) \\
\boldsymbol{\alpha}_{2}(\mathbf{x})
\end{array}\right] \\
& =\left[\begin{array}{c}
-L_{d}\left(c_{1} x_{1}+\frac{L_{q}}{L_{d}} p x_{2} x_{3}\right) \\
L_{q}\left(\begin{array}{c}
-\frac{p \varphi_{f}}{J_{m}} c_{2}\left(x_{2}-\omega_{\mathrm{ref}}\right)+\frac{R_{s}}{L_{q}} x_{3} \\
+\frac{L_{d}}{L_{q}} p x_{2} x_{1}+\frac{p \varphi_{f}}{L_{q}} x_{2} \\
-c_{3}\left(x_{3}-\frac{J_{m}}{p \varphi_{f}}\left(\frac{\varphi_{f}}{J_{m}} \omega_{\mathrm{ref}}+\frac{1}{J_{m}} T_{L}\right)\right)
\end{array}\right)
\end{array}\right],
\end{aligned}
$$

where $\omega_{\text {ref }}$ is the reference speed. It can be verified that disturbance-free system (3) can be stabilized by $\boldsymbol{\alpha}(\mathbf{x})$. Owing to its unimportance for the study of the paper, the derivation process of the controller for the disturbance-free system is omitted.

Let

$$
\begin{aligned}
\mathbf{V}(\mathbf{x})= & \frac{1}{2} x_{1}^{2}+\frac{1}{2} c_{2}\left(x_{2}-\omega_{\mathrm{ref}}\right)^{2} \\
& +\frac{1}{2}\left(x_{3}-\frac{J_{m}}{p \varphi_{f}}\left(\frac{\varphi_{f}}{J_{m}} \omega_{\mathrm{ref}}+\frac{1}{J_{m}} T_{L}\right)\right)^{2} .
\end{aligned}
$$

By means of some calculations and simplifications, we can get

$$
\begin{aligned}
\frac{\partial V(\mathbf{x})}{\partial \mathbf{x}}\left(\mathbf{f}(\mathbf{x})+\sum_{i=1}^{2} \mathbf{g}_{i}(\mathbf{x}) \boldsymbol{\alpha}_{i}\right) \\
=-\left(c_{1}+\frac{R_{s}}{L_{d}}\right) x_{1}^{2}-\frac{\varphi_{f}}{J_{m}} c_{2}\left(x_{2}-\omega_{\mathrm{ref}}\right)^{2} \\
\quad-c_{3}\left(x_{3}-\frac{J_{m}}{p \varphi_{f}}\left(\frac{\varphi_{f}}{J_{m}} \omega_{\mathrm{ref}}+\frac{1}{J_{m}} T_{L}\right)\right)^{2},
\end{aligned}
$$

$$
\begin{aligned}
& \frac{\partial \mathbf{V}(\mathbf{x})}{\partial \mathbf{x}} \sum_{i=1}^{m} \mathbf{g}_{i}(\mathbf{x}) \\
& \quad=\frac{1}{L_{d}} x_{1}+\frac{1}{L_{q}}\left(x_{3}-\frac{J_{m}}{p \varphi_{f}}\left(\frac{\varphi_{f}}{J_{m}} \omega_{\mathrm{ref}}+\frac{1}{J_{m}} T_{L}\right)\right) .
\end{aligned}
$$


To be more specific, the relevant parameters of the motor drive system are depicted as follows: the rated torque of motor $T_{e}=5.0 \mathrm{~N} \cdot \mathrm{m}$, number of rotor pole pairs $p=4$, flux linkage of permanent magnet $\varphi_{f}=0.18 \mathrm{~Wb}$, resistance of stator $R_{s}=$ $1.95 \Omega$, inductances of stator in $d$-axis and $q$-axis $L_{d}=L_{q}=$ $0.0115 \mathrm{H}$, moment of inertia of rotor $J_{m}=0.008 \mathrm{~kg} \cdot \mathrm{m}^{2}$, and damping coefficient of motor $B_{m}=0.01 \mathrm{~N} / \mathrm{rad} / \mathrm{s}$.

In addition, we assume $x^{\prime}=\left[x_{1},\left(x_{2}-\omega_{\text {ref }}\right),\left(x_{3}-\right.\right.$ $\left.\left.\left(J_{m} / p \varphi_{f}\right)\left(\left(\varphi_{f} / J_{m}\right) \omega_{\text {ref }}+\left(1 / J_{m}\right) T_{L}\right)\right)\right]^{T}$; by application of $(30)$ and (31) and selection of $c_{1}=8000, c_{2}=40$, and $c_{3}=8000$, we can get

$$
\begin{gathered}
\frac{1}{2}\left\|\mathbf{x}^{\prime}\right\|^{2} \leq \mathbf{V}(\mathbf{x}) \leq 20\left\|\mathbf{x}^{\prime}\right\|^{2}, \\
\frac{\partial V(\mathbf{x})}{\partial \mathbf{x}}\left(\mathbf{f}(\mathbf{x})+\sum_{i=1}^{2} \mathbf{g}_{i}(\mathbf{x}) \boldsymbol{\alpha}_{i}\right) \leq-7562\left\|\mathbf{x}^{\prime}\right\|^{2}, \\
\left|\frac{\partial \mathbf{V}(\mathbf{x})}{\partial \mathbf{x}} \sum_{i=1}^{m} \mathbf{g}_{i}(\mathbf{x})\right|^{2} \leq 7562\left\|\mathbf{x}^{\prime}\right\|^{2} .
\end{gathered}
$$

Hence, Assumption 1 is satisfied.

Let

$$
\begin{aligned}
& h_{1}(\mathbf{x})=\left[\begin{array}{ll}
0 & 3 L_{d} x_{1}
\end{array}\right]^{T}, \\
& h_{2}(\mathbf{x})=\left[\begin{array}{ll}
0 & 3 L_{q} x_{3}
\end{array}\right]^{T} .
\end{aligned}
$$

Hence,

$$
\begin{aligned}
& G_{1}=\frac{\partial h_{1}(\mathbf{x})}{\partial \mathbf{x}} \mathbf{g}_{1}(\mathbf{x})=\left[\begin{array}{ll}
0 & 3
\end{array}\right]^{T}, \\
& G_{2}=\frac{\partial h_{2}(\mathbf{x})}{\partial \mathbf{x}} \mathbf{g}_{2}(\mathbf{x})=\left[\begin{array}{ll}
0 & 3
\end{array}\right]^{T} .
\end{aligned}
$$

\section{Assumption 5 holds.}

Assume that $v_{1}$ and $v_{2}$ denote different nonlinear disturbance input signals and act on the currents of $d$-axis and $q$ axis, respectively. Choose $v_{1}=w_{1}$ and $v_{2}=w_{2}$. In other words, $v_{1}$ and $v_{2}$ represent that the system are immersed into two differently external disturbances.

For $v_{1}=w_{1}$, by means of some mathematical calculations, it can be obtained that

$$
\begin{aligned}
L_{A_{1} \mathbf{w}} \mathbf{v}_{1}(\mathbf{w}) & =\frac{\partial v_{1}(\mathbf{w})}{\partial \mathbf{w}} A_{1} \mathbf{w}=\left[\begin{array}{ll}
1 & 0
\end{array}\right]\left[\begin{array}{cc}
0 & 1 \\
-1 & 1
\end{array}\right]\left[\begin{array}{l}
w_{1} \\
w_{2}
\end{array}\right]=w_{2}, \\
L_{A_{1} \mathbf{w}}^{2} \mathbf{v}_{1}(\mathbf{w}) & =\frac{\partial L_{A_{1} \mathbf{w}} \mathbf{v}_{1}(\mathbf{w})}{\partial \mathbf{w}} A_{1} \mathbf{w}=\left[\begin{array}{ll}
0 & 1
\end{array}\right]\left[\begin{array}{cc}
0 & 1 \\
-1 & 1
\end{array}\right]\left[\begin{array}{l}
w_{1} \\
w_{2}
\end{array}\right] \\
& =-w_{1}+w_{2}=-a_{10} \mathbf{v}_{1}(\mathbf{w})+a_{11} L_{A_{1} \mathbf{w}} \mathbf{v}_{1}(\mathbf{w})
\end{aligned}
$$

Hence, there exist constants $r_{1}=2, a_{10}=-1$, and $a_{11}=1$ satisfying Assumption 4.
For $v_{2}=w_{2}$, by means of similar mathematical calculations, we can get

$$
\begin{aligned}
L_{A_{1} \mathbf{w}} \mathbf{v}_{2}(\mathbf{w}) & =\frac{\partial v_{2}(\mathbf{w})}{\partial \mathbf{w}} A_{1} \mathbf{w}=\left[\begin{array}{ll}
0 & 1
\end{array}\right]\left[\begin{array}{cc}
0 & 1 \\
-1 & 1
\end{array}\right]\left[\begin{array}{l}
w_{1} \\
w_{2}
\end{array}\right] \\
& =-w_{1}+w_{2}, \\
L_{A_{1} \mathbf{w}}^{2} \mathbf{v}_{2}(\mathbf{w}) & =\frac{\partial L_{A_{1} \mathbf{w}} \mathbf{v}_{2}(\mathbf{w})}{\partial \mathbf{w}} A_{1} \mathbf{w}=\left[\begin{array}{ll}
-1 & 1
\end{array}\right]\left[\begin{array}{cc}
0 & 1 \\
-1 & 1
\end{array}\right]\left[\begin{array}{l}
w_{1} \\
w_{2}
\end{array}\right] \\
& =-w_{1}=-a_{20} \mathbf{v}_{1}(\mathbf{w})+a_{21} L_{A_{1} \mathbf{w}} \mathbf{v}_{1}(\mathbf{w}) .
\end{aligned}
$$

Therefore, there exist constants $r_{1}=2, a_{20}=1$, and $a_{21}=1$ making Assumption 4 be satisfied.

In terms of (35), let

$$
\tau_{1}(\mathbf{w})=\operatorname{col}\left(\mathbf{v}_{1}(\mathbf{w}), L_{A_{1} \mathbf{w}} \mathbf{v}_{1}(\mathbf{w})\right)=\operatorname{col}\left(w_{1}, w_{2}\right)
$$

Then, there exist matrices $\Phi_{1}=\left[\begin{array}{cc}0_{\left(r_{1}-1\right) \times 1} & I_{r_{1}-1} \\ a_{10} & {\left[a_{11}, \ldots, a_{r_{1}-1}\right]}\end{array}\right]=$ $\left[\begin{array}{cc}0 & 1 \\ -1 & 1\end{array}\right]$ and $\psi_{1}=[1,0]$ making (11) hold and $\operatorname{rank}\left[\begin{array}{ll}\psi_{1} & \psi_{1} \Phi_{1}\end{array}\right]^{T}=2$.

According to (36), let

$$
\tau_{2}(\mathbf{w})=\operatorname{col}\left(\mathbf{v}_{2}(\mathbf{w}), L_{A_{1} \mathbf{w}} \mathbf{v}_{2}(\mathbf{w})\right)=\operatorname{col}\left(w_{2},-w_{1}+w_{2}\right) .
$$

In that way, there exist matrices $\Phi_{2}=$ $\left[\begin{array}{cc}0_{\left(r_{2}-1\right) \times 1} & I_{r_{2}-1} \\ a_{20} & {\left[a_{21}, \ldots, a_{r_{2}-1}\right]}\end{array}\right]=\left[\begin{array}{ll}0 & 1 \\ 1 & 1\end{array}\right]$ and $\psi_{2}=[1,0]$ making (11) satisfy and $\operatorname{rank}\left[\begin{array}{lll}\psi_{2} & \psi_{2} & \Phi_{2}\end{array}\right]^{T}=2$.

Choose $\Phi_{12}=A_{2}$; after some calculations it can be gotten that

$$
\frac{\partial \tau_{1}(\mathbf{w})}{\partial \mathbf{w}} A_{2} \mathbf{w}=\Phi_{12} \tau_{1}(\mathbf{w})
$$

Select $\Phi_{22}=\left[\begin{array}{ll}-1 & 0 \\ -1 & 0\end{array}\right]$; after some calculations we can get

$$
\frac{\partial \tau_{2}(\mathbf{w})}{\partial \mathbf{w}} A_{2} \mathbf{w}=\Phi_{22} \tau_{2}(\mathbf{w})
$$

Consequently, Assumption 7 is satisfied.

Assume $\widehat{\tau}_{1}(\mathbf{w})=T_{1} \tau_{1}(\mathbf{w})$, where $T_{1} \in R^{r_{1} \times r_{1}}$ is a nonsingular matrix, and by derivation of $\widehat{\tau}_{1}(\mathbf{w})$ along with (2), we can get

$$
\frac{\partial \widehat{\tau}_{1}(\mathbf{w})}{\partial \mathbf{w}} s_{1}(\mathbf{w})=T_{1} \phi_{1}(\mathbf{w}) T_{1}^{-1} \widehat{\tau}_{1}(\mathbf{w}),
$$

where $\phi_{1}(\mathbf{w})=\Phi_{1}+\varphi_{1}(\mathbf{w}), \varphi_{1}(\mathbf{w})=\Phi_{12} s_{12}(\mathbf{w})$.

Suppose $\widehat{\tau}_{2}(\mathbf{w})=T_{2} \tau_{2}(\mathbf{w})$, where $T_{2} \in R^{r_{2} \times r_{2}}$ is a nonsingular matrix and by derivation of $\widehat{\tau}_{2}(\mathbf{w})$ along with $(2)$, we can get

$$
\frac{\partial \widehat{\tau}_{2}(\mathbf{w})}{\partial \mathbf{w}} s_{2}(\mathbf{w})=T_{2} \phi_{2}(\mathbf{w}) T_{2}^{-1} \widehat{\tau}_{2}(\mathbf{w}),
$$

where $\phi_{2}(\mathbf{w})=\Phi_{2}+\varphi_{2}(\mathbf{w}), \varphi_{2}(\mathbf{w})=\Phi_{22} s_{22}(\mathbf{w})$. 

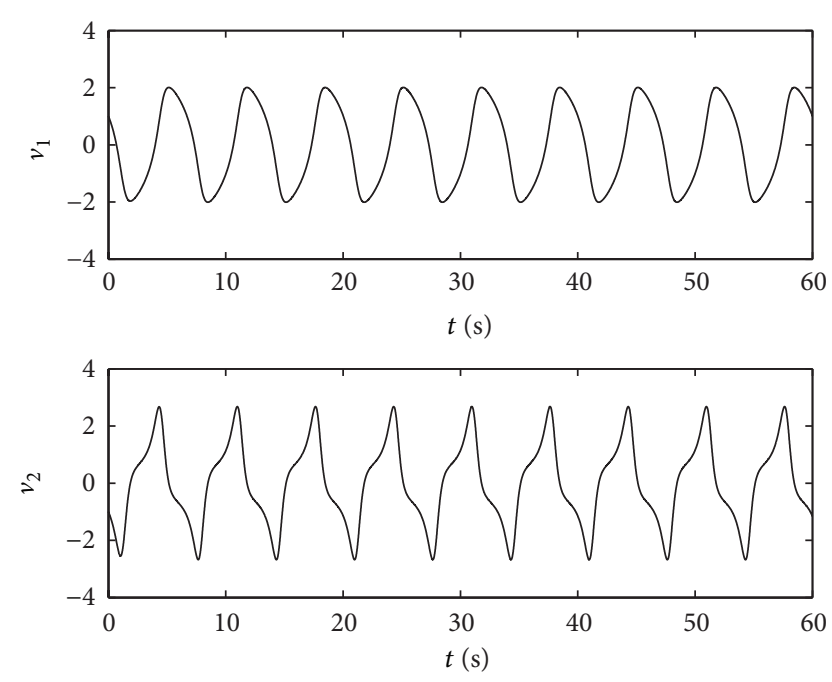

FIGURE 1: Multiclass nonlinear disturbance inputs.

Choose $F_{1}=\left[\begin{array}{cc}-2 & 1 \\ 0 & -6\end{array}\right]$; we can verify rank $\left[\begin{array}{ll}G_{1} & G_{1} F_{1}\end{array}\right]^{T}=2$. Solving Sylvester equation $T_{1} \Phi_{1}-F_{1} T_{1}=G_{1} \psi_{1}$, it can be gotten that

$$
T_{1}=\left[\begin{array}{ll}
0.1993 & -0.0897 \\
0.4884 & -0.0698
\end{array}\right] \text {. }
$$

Select $F_{2}=\left[\begin{array}{cc}-2 & 1 \\ 0 & -6\end{array}\right]$. Similarly, we are able to obtain $\operatorname{rank}\left[\begin{array}{lll}G_{2} & G_{2} F_{2}\end{array}\right]^{T}=2$. Solving Sylvester equation $T_{2} \Phi_{2}-$ $F_{2} T_{2}=G_{2} \psi_{2}$, we can get

$$
T_{2}=\left[\begin{array}{ll}
0.3220 & -0.1317 \\
0.5122 & -0.0732
\end{array}\right] .
$$

It has already been verified that all assumed conditions required for systems (27) and (6) are satisfied. Following the design procedure given in Sections 3 and 4, the corresponding nonlinear internal models and state feedback controller are able to be designed as below:

$$
\begin{aligned}
\dot{\xi}_{1}= & \left(-2-1.4651 w_{1}^{2}\right) \xi_{1}+\left(1+0.598 w_{1}^{2}\right)\left(\xi_{2}+0.0345 x_{1}\right), \\
\dot{\xi}_{2}= & -1.1395 w_{1}^{2} \xi_{1}+\left(-6+0.4651 w_{1}^{2}\right)\left(\xi_{2}+0.0345 x_{1}\right) \\
& +5.85 x_{1}-0.138 x_{2} x_{3}-3 u_{1}, \\
\dot{\xi}_{3}= & \left(-2+0.3171 w_{1}^{2}\right) \xi_{3}+\left(1-0.5707 w_{1}^{2}\right)\left(\xi_{4}+0.0345 x_{1}\right), \\
\dot{\xi}_{4}= & 0.7317 w_{1}^{2} \xi_{3}+\left(-6-1.3171 w_{1}^{2}\right)\left(\xi_{4}+0.0345 x_{1}\right) \\
& -5.85 x_{3}-0.138 x_{2} x_{1}-2.16 x_{2}+3 u_{2}, \\
u_{1}= & -92.046 x_{1}-0.046 x_{2} x_{3}+2.3333 \xi_{1}-3 \xi_{2}, \\
u_{2}= & -413.28 x_{2}-90.096 x_{3}+249550+0.046 x_{2} x_{1} \\
& +127.7778 T_{L}+1.6667 \xi_{3}-3 \xi_{4} .
\end{aligned}
$$

In order to evaluate the validity of the proposed method, the numerical simulations are performed in Matlab software
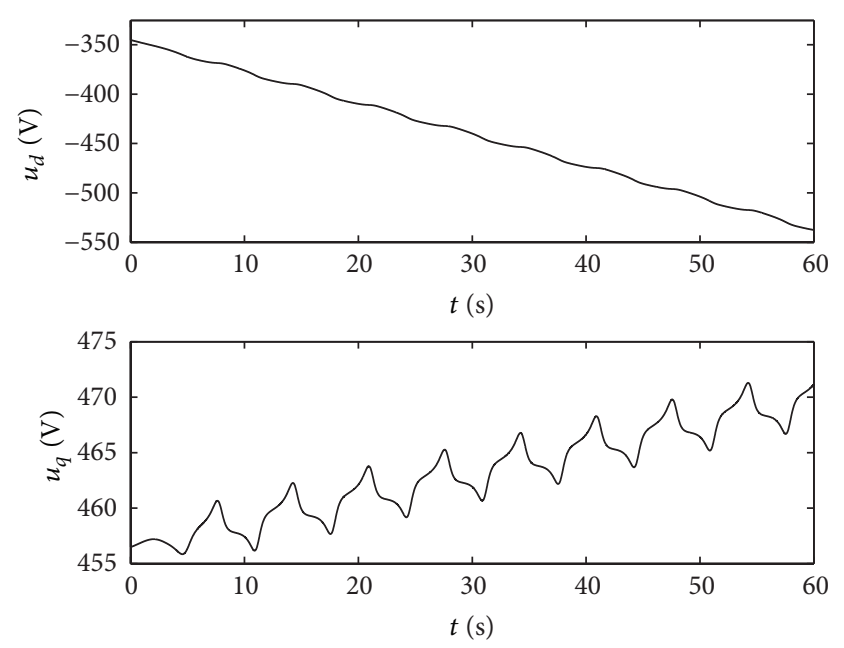

FIGURE 2: System control inputs.

with the sampling interval $\Delta t=0.001 \mathrm{~s}$ and initial conditions $x(0)=\left[\begin{array}{lll}0.1 & 0 & 1.0\end{array}\right], \widehat{\eta}(0)=\left[\begin{array}{llll}0 & 0 & 0 & 0\end{array}\right]$, and $\mathrm{w}(0)=$ $\left[\begin{array}{ll}1 & -1\end{array}\right]$. In addition, the reference values of $d$-axis current $i_{d}$ and speed $\omega_{m}$ are set to zero and $600 \mathrm{r} / \mathrm{min}$, respectively, and $q$-axis current $i_{q}$ tracks the linear variation value of load $T_{L}$. Ultimately, the simulation results are depicted in Figures 1, 2, and 3. Figure 1 describes the multiclass nonlinear nonharmonic disturbances $v_{1}$ and $v_{2}$, in which we can see that the exogenous disturbances immersing into the system are bounded and different, and they can originate from different exosystems so long as the exosystems can be written in the form of (2). Figure 2 demonstrates the control inputs $u_{d}$ and $u_{q}$, which matches the external disturbances and reference values; and their combined actions make the different disturbances be rejected and ensure the system outputs to track the reference values. Figure 3 indicates the state outputs of the system, in which we can see that the $d$-axis current $i_{d}$ and speed $\omega_{m}$ are able to track their own reference values in high precision. In other words, the simulation results signify that the proposed algorithm is able to reject the multiclass external disturbance effectively and ensures that the closed-loop system rapidly converge to the reference values. In consequence, the designed internal models can replicate with the different exogenous disturbances and the proposed controller has an excellent control performance.

\section{Conclusions}

In the paper, an IMP based multivariable nonlinear control algorithm with multiclass nonharmonic disturbances is proposed to suppress the differently external nonharmonic disturbances and control the closed-loop system to track the reference values. The major conclusions of the research are summarized as follows:

(1) in light of the multiclass nonharmonic disturbances, a class of new multiclass nonlinear internal models can be constructed on the basis of the definitions of expanded steady state generator and IMP, and 

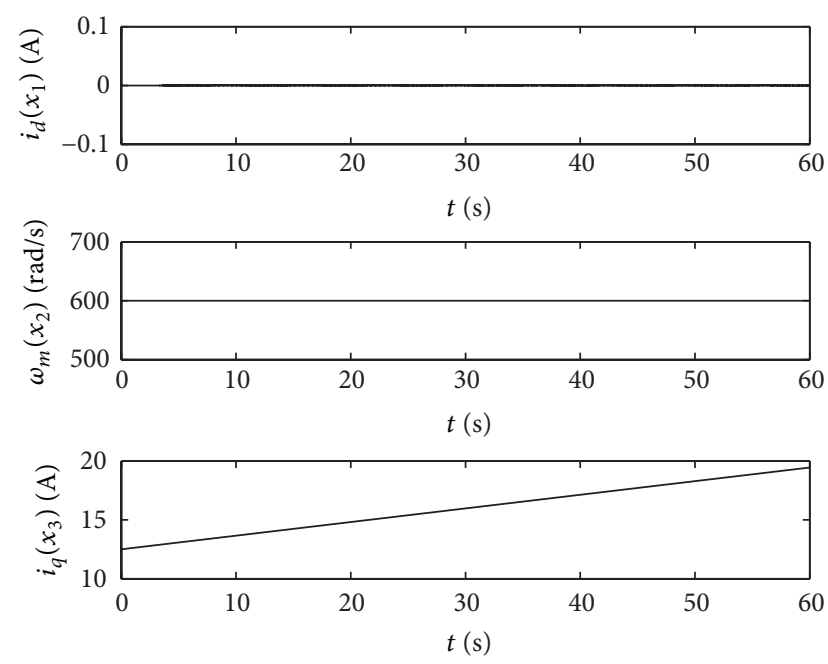

FIGURE 3: System state outputs.

the characteristics of which depend on the structural information of exosystem and state information of closed-loop system;

(2) a multivariable nonlinear state feedback controller associated with the designed internal model equations is designed; the application of Lyapunov theory can justify the convergence of the proposed control algorithm;

(3) the application of the algorithm into a two-input variable motor drive system demonstrates that the presented algorithm is able to suppress the multiclass disturbances and guarantee the multivariable closedloop system global uniform convergence.

\section{Conflict of Interests}

The authors declare that there is no conflict of interests regarding the publication of this paper.

\section{Acknowledgments}

This work was supported by the Specialized Research Fund for the Doctoral Program of Higher Education of China (Grant no. 20120036130001), the Fundamental Research Funds for the Central Universities of China (Grant no. 2014MS93), and the Independent Research Funds of State Key Laboratory of Alternate Electrical Power System with Renewable Energy Sources of China (Grant no. 201414).

\section{References}

[1] B. A. Francis and W. M. Wonham, "The internal model principle for linear multivariable regulators," Applied Mathematics and Optimization, vol. 2, no. 2, pp. 170-194, 1975.

[2] C. Commault, J.-M. Dion, and A. Perez, "Disturbance rejection for structured systems," IEEE Transactions on Automatic Control, vol. 36, no. 7, pp. 884-887, 1991.
[3] R. Saeks and J. Murray, "Feedback system design: the tracking and disturbance rejection problems," IEEE Transactions on Automatic Control, vol. 26, no. 1, pp. 203-217, 1981.

[4] A. Isidori and C. I. Byrnes, "Output regulation of nonlinear systems," IEEE Transactions on Automatic Control, vol. 35, no. 2, pp. 131-140, 1990.

[5] Y. Jiang and S. Liu, "Global disturbance rejection of a class of nonlinear Systems with unknown exosystems," Journal of Control Theory and Applications, vol. 7, no. 4, pp. 379-383, 2009.

[6] J. Huang and Z. Chen, "A general framework for tackling the output regulation problem," IEEE Transactions on Automatic Control, vol. 49, no. 12, pp. 2203-2218, 2004.

[7] G. Wang and H. Karimi, "Data-driven design of robust fault detection system for wind turbines," Mechatronics, vol. 24, no. 4, pp. 298-306, 2014.

[8] S. Ding, X. Xie, and H. Luo, "A review on basic data-driven approaches for industrial process monitoring," IEEE Transactions on Industrial Electronics, no. 99, 1 page, 2014.

[9] S. Yin, X. Li, H. Gao, and O. Kaynak, "Data-based techniques focused on modern industry: an overview," IEEE Transactions on Industrial Electronics, no. 99, 1 page, 2014.

[10] T. C. S. Hsia, "A new technique for robust control of servo systems," IEEE Transactions on Industrial Electronics, vol. 36, no. 1, pp. 1-7, 1989.

[11] Q. Jia, S. Cao, G. Guo, and J. Yu, "External disturbance rejection by use of an add-on nonlinear controller in HDD servo systems," IEEE Transactions on Magnetics, vol. 49, no. 6, pp. 2624-2627, 2013.

[12] S. Yin, S. Ding, A. Haghani, H. Hao, and P. Zhang, "A comparison study of basic data-driven fault diagnosis and process monitoring methods on the benchmark Tennessee Eastman process," Journal of Process Control, vol. 22, no. 9, pp. 1567-1581, 2012.

[13] S. Yin, S. Ding, A. Haghani, and H. Hao, "Data-driven monitoring for stochastic systems and its application on batch process," International Journal of Systems Science, vol. 44, no. 7, pp. 13661376, 2013.

[14] L. Paunonen and S. Pohjolainen, "Reduced order internal models in robust output regulation," IEEE Transactions on Automatic Control, vol. 58, no. 9, pp. 2307-2318, 2013.

[15] A. Isidori and C. I. Byrnes, "Output regulation of nonlinear systems," IEEE Transactions on Automatic Control, vol. 35, no. 2, pp. 131-140, 1990.

[16] J. Huang and W. J. Rugh, "On a nonlinear multivariable servomechanism problem," Automatica, vol. 26, no. 6, pp. $963-$ 972, 1990.

[17] Z. Ding, "Asymptotic rejection of unknown sinusoidal disturbances in nonlinear systems," Automatica, vol. 43, no. 1, pp. 174$177,2007$.

[18] A. Serrani, A. Isidori, and L. Marconi, "Semiglobal nonlinear output regulation with adaptive internal model," IEEE Transactions on Automatic Control, vol. 46, no. 8, pp. 1178-1194, 2001.

[19] Z. Ding, "Adaptive estimation and rejection of unknown sinusoidal disturbances in a class of non-minimum-phase nonlinear systems," IEE Proceedings: Control Theory and Applications, vol. 153, no. 4, pp. 379-386, 2006.

[20] Y. Jiang and S. T. Liu, "Rejection of nonharmonic disturbances in a class of nonlinear systems with nonlinear exosystems," Asian Journal of Control, vol. 13, no. 6, pp. 858-867, 2011.

[21] C. L. Chen, Z. Ding, and B. Lennox, "Rejection of nonharmonic disturbances in nonlinear systems with semi-global stability," 
IEEE Transactions on Circuits and Systems II: Express Briefs, vol. 55, no. 12, pp. 1289-1293, 2008.

[22] J. Huang and W. J. Rugh, "On a nonlinear multivariable servomechanism problem," Automatica, vol. 26, no. 6, pp. 963972, 1990

[23] Y. Yu, Z. Q. Mi, Y. K. Xu, and T. Zhao, "Global multivariable control of permanent magnet synchronous motor for mechanical elastic energy storage system under multiclass nonharmonic external disturbances," Abstract and Applied Analysis, vol. 2014, Article ID 410282, 9 pages, 2014.

[24] Z. Y. Chen and J. Huang, "Robust output regulation with nonlinear exosystems," Automatica, vol. 41, no. 8, pp. 1447-1454, 2005.

[25] Z. Ping and J. Huang, "Global robust output regulation for a class of multivariable systems and its application to a motor drive system," in Proceedings of the American Control Conference (ACC '11), pp. 4560-4565, San Francisco, Calif, USA, July 2011. 


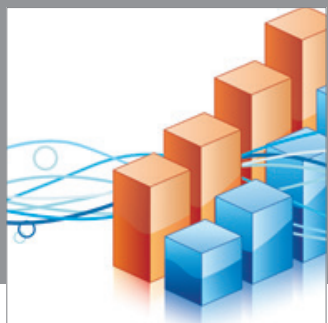

Advances in

Operations Research

mansans

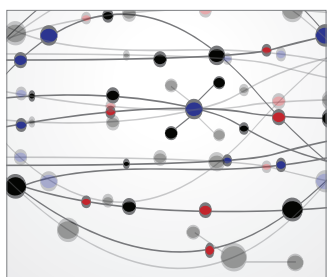

The Scientific World Journal
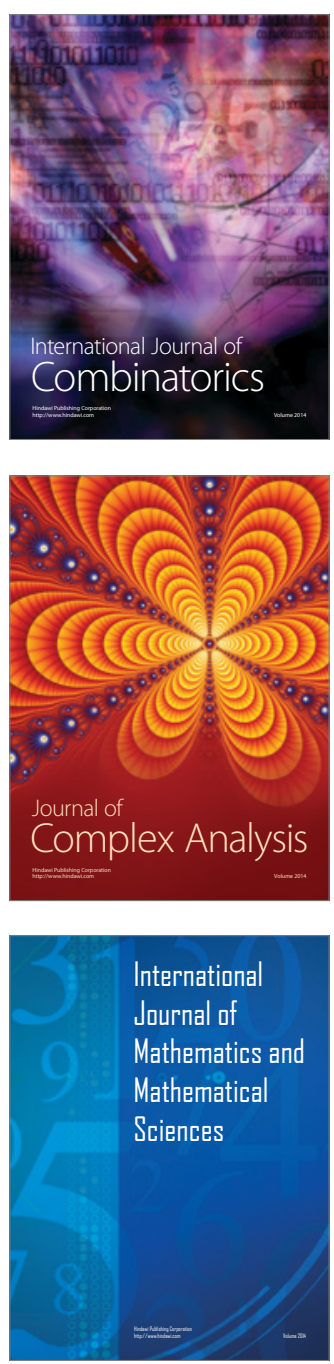
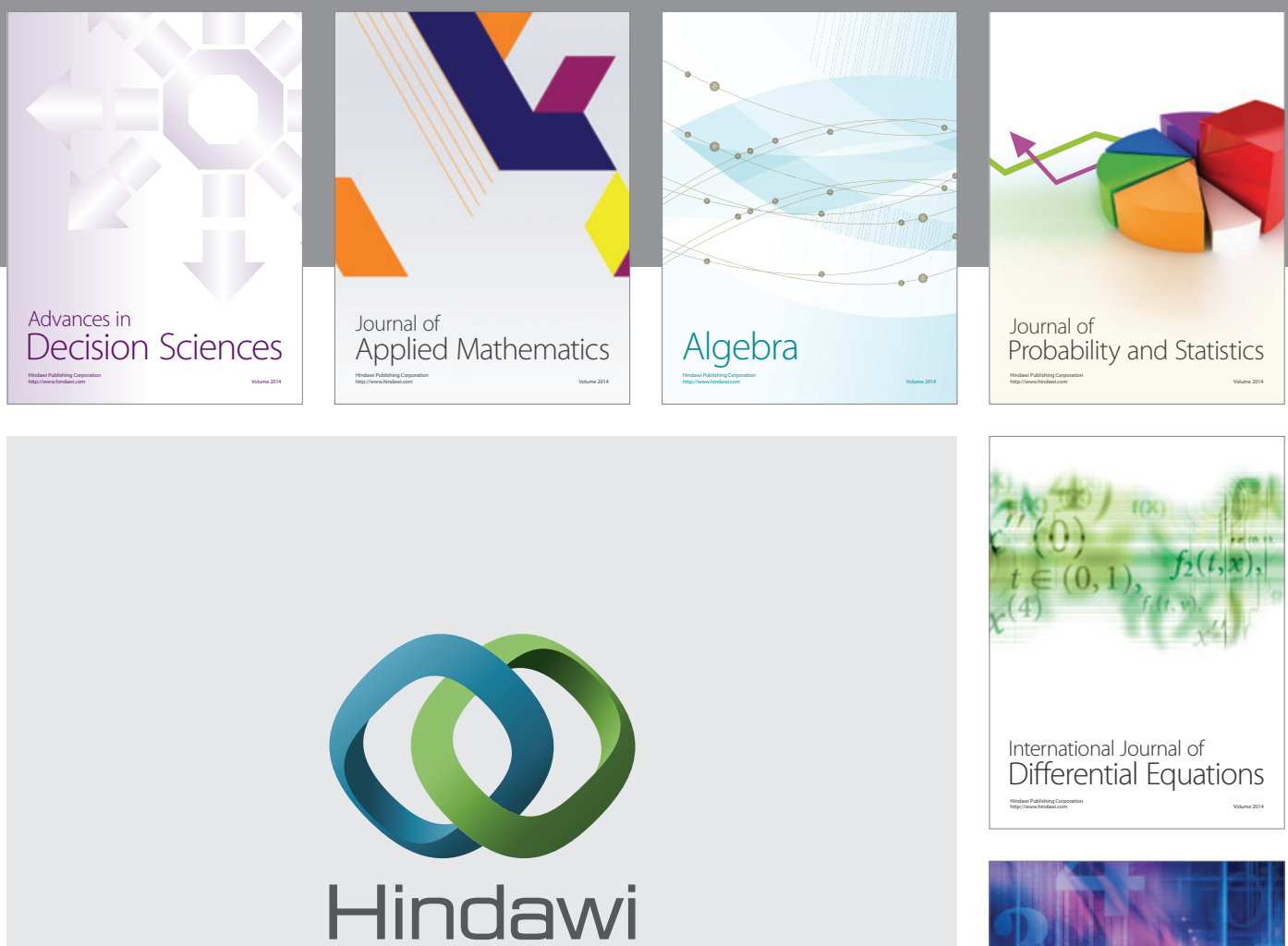

Submit your manuscripts at http://www.hindawi.com
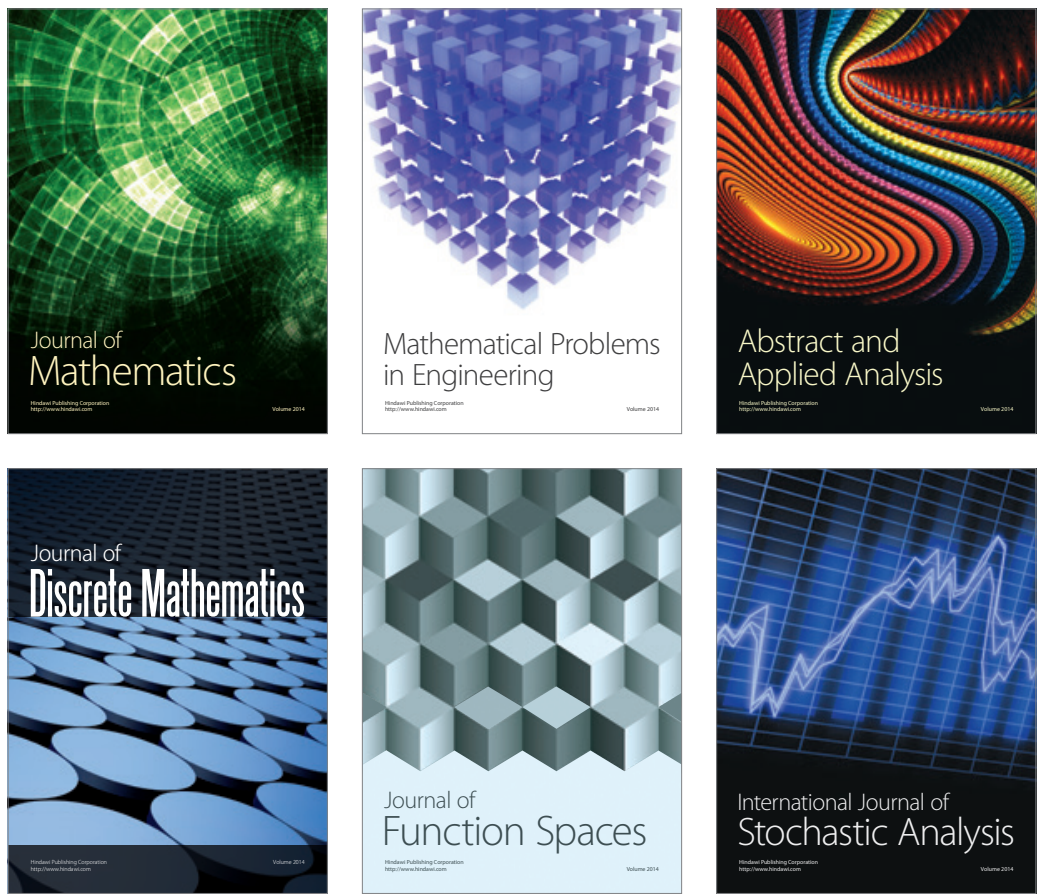

Journal of

Function Spaces

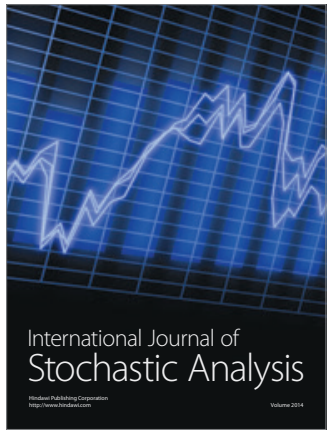

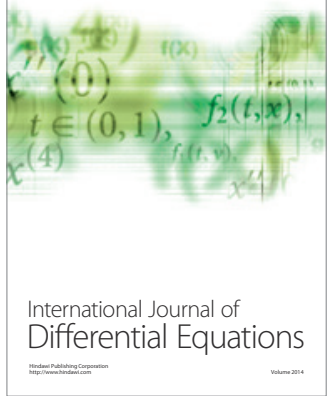
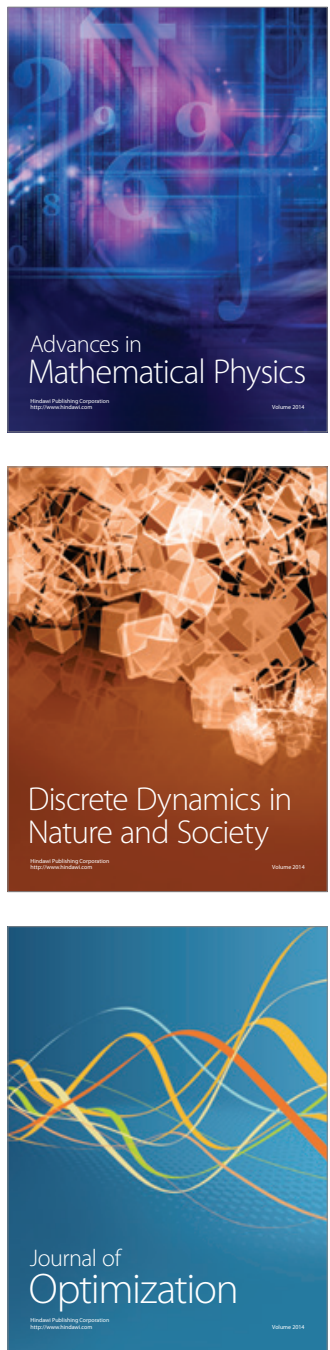